\title{
Perioperative Management of Two Pediatric Patients With Hypertrophic Cardiomyopathy Undergoing Minimally Invasive Surgical Procedures
}

\author{
Sathappan Karuppiah ${ }^{\mathrm{a}}$, Ahsan Syed ${ }^{\mathrm{a}, \mathrm{b}}$, Aymen Naguib ${ }^{\mathrm{a}, \mathrm{b}}$, Joseph D. Tobiasa, b, c
}

\begin{abstract}
Hypertrophic cardiomyopathy (HCM) is the most common genetic disease of the heart and remains an important etiology of sudden cardiac death in adolescents and young adults. Autosomal dominant inheritance of a mutation in one of the numerous genes encoding for proteins in the myocardial sarcomere leads to an abnormal myocyte growth pattern with hypertrophy. Perioperative management of patients with HCM for any type of surgical procedure carries an increased risk of morbidity and mortality related to the propensity for arrhythmias and myocardial ischemia. During the perioperative period, myocardial function may be impacted by alterations in preload, afterload, and myocardial contractility related to the surgical procedure, anesthetic agents, and changes in intravascular volume. We present two pediatric patients with a known diagnosis of HCM who presented for non-cardiac, minimally invasive surgical (MIS) procedures. The perioperative implications of HCM are reviewed with particular emphasis placed on the potential cardiovascular alterations induced by the MIS procedure with increases in intra-thoracic or intra-abdominal pressure.
\end{abstract}

Keywords: Hypertrophic cardiomyopathy; Minimally invasive surgeries; Perioperative management

\section{Introduction}

Hypertrophic cardiomyopathy (HCM) is characterized by asymmetric or symmetric hypertrophy of the interventricular septum (IVS), left ventricle (LV), or right ventricle (RV), resulting in impaired diastolic filling and mitral valve abnormali-

Manuscript accepted for publication January 08, 2016

aDepartment of Anesthesiology \& Pain Medicine, Nationwide Children's Hospital, Columbus, $\mathrm{OH}$, USA

${ }^{b}$ Department of Anesthesiology \& Pain Medicine, The Ohio State University College of Medicine, Columbus, OH, USA

${ }^{\mathrm{c}}$ Corresponding Author: Joseph D. Tobias, Department of Anesthesiology \& Pain Medicine, Nationwide Children's Hospital, 700 Children's Drive, Columbus, OH 43205, USA. Email: Joseph.Tobias@Nationwidechildrens.org

doi: http://dx.doi.org/10.14740/jmc2410w ties with or without intermittent left ventricular outflow tract (LVOT) obstruction [1,2]. It was first reported by Brock and Teare in 1957 and 1958 as subaortic stenosis and asymmetrical septal hypertrophy, respectively $[3,4]$. HCM has an incidence of $0.2 \%$, is the most common genetic disease of heart, and remains a common etiology of sudden cardiac death in young adults and athletes $[5,6]$. It is inherited as an autosomal dominant disorder, characterized by a mutation in one of the numerous genes encoding for proteins in the myocardial sarcomere, leading to an abnormal myocyte growth pattern [7-9]. Although the nomenclature has varied over the years, HCM is now used to classify LV hypertrophy with a non-dilated ventricular chamber without a cardiac or systemic disease causing the hypertrophy $[10,11]$.

Perioperative management of patients with HCM for any type of surgical procedure carries an increased risk of morbidity and mortality related to the propensity for arrhythmias and myocardial ischemia [12-14]. Furthermore, myocardial function may be impacted by alterations in preload, afterload, and myocardial contractility related to the surgical procedure, anesthetic agents, and changes in intravascular volume. We present two pediatric patients with a known diagnosis of HCM who presented for non-cardiac, minimally invasive surgical (MIS) procedures. The perioperative implications of $\mathrm{HCM}$ are reviewed with particular emphasis placed on the potential cardiovascular alterations induced by the MIS procedure with increases in intra-thoracic or intra-abdominal pressure (IAP).

\section{Case Reports}

IRB approval at Nationwide Children's Hospital is not required for case reports involving two or fewer patients.

\section{Case 1}

A 10-year-old, $39.6 \mathrm{~kg}$ boy with severe HCM and an automated implantable cardioverter defibrillator (AICD) in situ presented with noisy breathing and dyspnea on exertion (NYHA class III) for several weeks. There were no complaints of coughing, wheezing or increased work of breathing. His family history was suggestive of HCM in his maternal aunt and grandfather. His medication regimen included atenolol $25 \mathrm{mg}$ 
by mouth twice daily, albuterol inhaler as needed, and montelukast chewable tablet. On physical examination, his vitals were stable with a grade III systolic ejection murmur heard at the left lower sternal border which increased on standing with a Valsalva maneuver. Laboratory evaluation prior to the surgery showed hemoglobin of $11.6 \mathrm{~g} / \mathrm{dL}$, hematocrit $35.8 \%$, and a platelet count of $175,000 / \mathrm{mm}^{3}$. Coagulation studies, serum electrolytes and blood glucose levels were within normal limits. Electrocardiogram (ECG) revealed left ventricular hypertrophy by voltage criteria with a normal sinus rhythm. Transthoracic echocardiogram (TTE) showed asymmetrical septal hypertrophy with a gradient of $25 \mathrm{~mm} \mathrm{Hg}$ with hyperdynamic right and left ventricular function, a dilated left atrium, thickened mitral valve, moderate mitral regurgitation and dynamic LVOT obstruction. Computerized tomography (CT) of the chest showed bulky lymphadenopathy in the upper right mediastinum deviating the trachea to the left in association with a right upper lobe nodular density measuring $3.2 \times$ $3.4 \mathrm{~cm}$. There were ground glass opacities of the lung fields and nodules concerning for malignancy or infection. He was scheduled for elective video-assisted thoracoscopic (VATS) biopsy under general anesthesia. The patient was held nil per os for $8 \mathrm{~h}$ except for his usual medications, the AICD was inactivated for the surgical procedure, and a peripheral intravenous infusion was started to provide maintenance intravenous fluids. Premedication included intravenous midazolam (2 mg). The patient was transported to the operating room and routine American Society of Anesthesiologists' (ASA) monitors were placed. Anesthesia was induced with etomidate $(0.3 \mathrm{mg} /$ $\mathrm{kg})$, fentanyl $(5 \mu \mathrm{g} / \mathrm{kg})$, and lidocaine $(1 \mathrm{mg} / \mathrm{kg})$. Neuromuscular blockade was provided by rocuroniuim $(1 \mathrm{mg} / \mathrm{kg})$. Under direct laryngoscopy, a 28-French, left-sided double lumen tube (DLT) was placed and position was confirmed by use of fiberoptic bronchoscopy. A second peripheral intravenous cannula and a left radial arterial cannula were placed. The patient was positioned with his right side up and one-lung ventilation (OLV) was initiated to maintain normocarbia. Anesthesia was maintained with a dexmedetomidine infusion at $0.2-0.4 \mu \mathrm{g} /$ $\mathrm{kg} / \mathrm{h}$ without a loading dose and inhaled sevoflurane $(0.5-2 \%)$ in air and oxygen to maintain the oxygen saturation at $94-96 \%$. During the VATS procedure, effective lung isolation with OLV was noted. Wedge biopsy of the RUL nodule and lymph node were obtained and sent for frozen pathological examination to rule out malignancy. A necrotizing granuloma was noted, suggestive of histoplasmosis. The hemodynamic parameters remained stable throughout the procedure with a heart rate ranging from 60 to 80 beats/min and a systolic blood pressure (BP) from 90 to $120 \mathrm{~mm} \mathrm{Hg}$. Hypotension (systolic BP less than 80 $\mathrm{mm} \mathrm{Hg}$ ) was treated with a fluid bolus and phenylephrine (1 $\mu \mathrm{g} / \mathrm{kg})$. The procedure lasted approximately $2.5 \mathrm{~h}$. Fluid administration included $900 \mathrm{~mL}$ of crystalloid and $500 \mathrm{~mL}$ of $5 \%$ albumin. After the biopsy was obtained, two-lung ventilation was resumed. Arterial blood gas analysis at the completion of the procedure was unremarkable with hemoglobin of $10.5 \mathrm{~g} /$ $\mathrm{dL}$, a hematocrit of $31 \%$, and normal electrolytes and blood glucose values. At the completion of the surgical procedure, neuromuscular block was reversed with standard doses of neostigmine and glycopyrrolate and the patient's trachea was extubated. The patient was admitted to the cardiothoracic sur- gical intensive care (CTICU) for overnight monitoring. Postoperative pain was managed with intravenous acetaminophen $(15 \mathrm{mg} / \mathrm{kg}$ ) every $6 \mathrm{~h}$ and patient-controlled analgesia with hydromorphone. His postoperative period was unremarkable and he was transferred to the inpatient floor on postoperative day (POD) 2 and discharged home on POD 4.

\section{Case 2}

A 14-year-old, $32 \mathrm{~kg}$ boy with non-obstructed HCM with a history of incomplete bladder emptying, posterior urethral valves, and hydronephrosis with renal insufficiency presented for robotic-assisted laparoscopic appendicovesicostomy. The patient also had a history of hypertension, previous placement of a urethral stent, and renal insufficiency secondary to obstructive uropathy. There was no family history of cardiomyopathy. His medication regimen included oral lisinopril $5 \mathrm{mg}$ once a day and calcitriol $0.5 \mu \mathrm{g}$ once daily. On physical examination, his vital signs were stable with a grade IV systolic ejection murmur heard at the left lower sternal border which increased on standing with a Valsalva maneuver. Laboratory evaluation prior to the surgery showed a hemoglobin of $12 \mathrm{~g} / \mathrm{dL}$, hematocrit $36.5 \%$, and a platelet count of $292,000 / \mathrm{mm}^{3}$. Coagulation studies, serum electrolytes, and blood glucose levels were within normal limits. Renal function test showed an elevated creatinine and blood urea nitrogen (BUN) of 2.85 and $27 \mathrm{mg} /$ $\mathrm{dL}$, respectively. ECG and 24-h Holter study revealed a normal sinus rhythm without evidence of arrhythmias. TTE showed asymmetrical septal hypertrophy with normal chamber sizes and normal ventricular function with no LVOT obstruction. Renal ultrasound showed bilateral echogenic kidneys with cortical scarring and moderate hydronephrosis on the left side.

The patient was held nil per os for $8 \mathrm{~h}$ except for his usual medications and a peripheral intravenous infusion was started to provide maintenance intravenous fluids. Premedication included intravenous midazolam $(1.5 \mathrm{mg})$. The patient was transported to the operating room and routine ASA monitors were placed. Anesthesia was induced with propofol $3 \mathrm{mg} / \mathrm{kg}$, fentanyl $(2 \mu \mathrm{g} / \mathrm{kg})$, and lidocaine $(1 \mathrm{mg} / \mathrm{kg})$. Neuromuscular blockade was provided by cis-atracurium $(0.2 \mathrm{mg} / \mathrm{kg})$. Under direct laryngoscopy, the trachea was intubated with a $6.0 \mathrm{~mm}$ cuffed endotracheal tube. A second peripheral intravenous cannula and a right radial arterial cannula were placed. Anesthesia was maintained with a dexmedetomidine infusion at $0.2-0.4$ $\mu \mathrm{g} / \mathrm{kg} / \mathrm{h}$ without a loading dose, remifentanil infusion $0.2-0.3$ $\mu \mathrm{g} / \mathrm{kg} / \mathrm{min}$ and inhaled desflurane (4-5\%) in air and oxygen to maintain the oxygen saturation at $94-96 \%$. During the roboticassisted laparoscopic procedure, the abdomen was insufflated to a maximum IAP of $12 \mathrm{~cm} \mathrm{H}_{2} \mathrm{O}$. The hemodynamic parameters remained stable throughout the procedure with a heart rate ranging from 80 to 100 beats $/ \mathrm{min}$ and a mean $\mathrm{BP}$ from 60 to $70 \mathrm{~mm} \mathrm{Hg}$. Hypotension (systolic BP less than $80 \mathrm{~mm}$ $\mathrm{Hg}$ ) was treated with a fluid bolus $(10 \mathrm{~mL} / \mathrm{kg}$ of $5 \%$ albumin) and phenylephrine $(1 \mu \mathrm{g} / \mathrm{kg})$ as needed. The procedure lasted approximately $3 \mathrm{~h}$. Fluid administration included $850 \mathrm{~mL}$ of crystalloid and $250 \mathrm{~mL}$ of $5 \%$ albumin. Arterial blood gas analysis at the completion of the procedure showed a hemoglobin of $9.2 \mathrm{~g} / \mathrm{dL}$, a hematocrit of $27 \%$, lactate $0.5 \mathrm{mM} / \mathrm{L}$ and 
normal electrolytes and blood glucose values. Neuromuscular block was reversed with standard doses of neostigmine and glycopyrrolate and the patient's trachea was extubated. The patient was admitted to the CTICU for overnight monitoring. Postoperative pain was managed with intravenous acetaminophen $(15 \mathrm{mg} / \mathrm{kg})$ every $6 \mathrm{~h}$ and patient-controlled analgesia with hydromorphone. His postoperative course was unremarkable and he was transferred to the inpatient floor on POD 2 and discharged home on POD 4.

\section{Discussion}

HCM is generally diagnosed when the patient or a close relative manifests clinical signs and symptoms such as chest pain, arrhythmias or sudden cardiac death. The most common echocardiographic finding is a hyperdynamic LV with diastolic dysfunction and abnormal ventricular compliance. Arrhythmias are common and often intractable in HCM as noted in our first patient who required placement of an AICD. The arrhythmias may be of atrial or ventricular origin, frequently altering ventricular preload and impacting hemodynamic function. Preoperatively, if there are clinical concerns regarding arrhythmias or findings on the preoperative ECG, more thorough investigations including 24-h Holter monitoring or electrophysiological studies may be indicated prior to anesthetic care.

Although there are limited data for the pediatric-aged patient, $40 \%$ of adult HCM patients have at least one adverse cardiac event during non-cardiac surgery [8]. Predictors of adverse perioperative cardiac event (congestive heart failure, myocardial ischemia and dysrhythmias) include the magnitude of the surgical procedure and the duration of surgery. The degree of the resting LVOT gradient, systolic anterior motion of the mitral valve, severity of mitral regurgitation, the presence of left ventricular dysfunction, and septal thickness did not correlate with the incidence of perioperative adverse cardiac events. Given the complex nature of our patients' co-morbid cardiac disease, close collaboration with our pediatric cardiology colleagues was required. This included not only noninvasive imaging, but also preoperative discussion regarding the optimal perioperative management.

The goals of perioperative care for patients with HCM are to limit the dynamic consequences of HCM with avoidance of tachycardia, maintenance of normal sinus rhythm, normal to slightly decreased myocardial contractility, while maintaining baseline preload and afterload. Control of these hemodynamic factors limits the increase in the outflow tract gradient thereby maintaining cardiac output. While many outpatient pediatric patients are held nil per os for several hours prior to anesthetic care, in patients with significant myocardial involvement, long periods of fasting may not be tolerated and hence intravenous fluids should be considered during nil per os times. Additionally, the presence of intravenous access allows for the rapid administration of resuscitative medications or fluids when such hemodynamic alterations occur during anesthetic induction.

Hemodynamic fluctuations with alterations in contractility, preload and afterload may be particularly common during anesthetic induction, direct laryngoscopy, and endotracheal intubation. In our first patient, we chose etomidate, fentanyl and lidocaine given their hemodynamic stability. Etomidate has been shown in multiple studies to have stable hemodynamic properties in both adult and pediatric patients, even in the presence of significant myocardial involvement $[15,16]$. Although its adrenal suppressive effects due to inhibition of $11-\beta$ hydroxylase have made its use controversial in specific clinical scenarios, adverse perioperative implications of this effect have not been clearly demonstrated $[17,18]$. In our second patient, as his hemodynamic status was judged to be more stable, intravenous propofol and fentanyl were used for anesthetic induction.

For maintenance anesthesia in both patients, we chose a combination of dexmedetomidine and sevoflurane. Of the available inhalational anesthetic agents (sevoflurane, isoflurane and desflurane), sevoflurane has the least effect on systemic vascular resistance while having negative chronotropic effects, both of which may be beneficial in patients with HCM. The dose requirements for sevoflurane were decreased by the concomitant administration of dexmedetomidine which also have negative chronotropic properties and may have anti-arrhythmic properties during the perioperative period [19]. Additionally, we chose dexmedetomidine to supplement maintenance anesthesia in our first patient as it has limited effects on oxygenation during OLV [20]. Although hypotension may be seen with dexmedetomidine, it provides effective sympatholysis thereby effectively controlling heart rate and myocardial contractility. As noted in both of our patients, the incidence of hypotension with dexmedetomidine can be limited by omitting the loading dose. Control of the perioperative hemodynamic parameters (maintenance of a low heart rate and a high-normal systemic vascular resistance) was facilitated by the intermittent use of intraoperative phenylephrine to treat hypotension. An additional concern perioperatively with HCM is the potential for an imbalance of the myocardial oxygen deliverydemand ratio. Histologically, the thickened walls of HCM may result in luminal narrowing of the intramural coronary arteries [21]. As such, diastolic BP should be maintained and heart rate should be controlled.

Given the associated co-morbid cardiac disease and the magnitude of the surgical procedure in our two patients, intraoperative monitoring included invasive monitoring of arterial BP to ensure that we could quickly identify and treat hypotension. For more invasive procedures where significant alterations in hemodynamic parameters are expected or if unexplained intraoperative hemodynamic instability occurs, central venous pressure (CVP) monitoring or intraoperative TEE may be warranted. As such, we would recommend the ready availability of a pediatric cardiologist for intraoperative consultation. In addition to TEE and CVP monitoring, mixed-venous oxygen saturation (SvO2) monitoring and periodic evaluation of plasma lactate concentration may be helpful in evaluating end-organ oxygen delivery [22-25]. Alternatively, near-infrared spectroscopy may be used to monitor cerebral and tissue oxygenation as an indicator of end organ perfusion [26].

Intraoperative care in our patients was also impacted by the associated MIS procedure using VATS and laparoscopy $[27,28]$. Insufflation into the thoracic or abdomen can have adverse physiologic effects including decreased preload, increases afterload, and increased pulmonary vascular resistance. For 
minimally invasive thoracic procedures, the effective use of OLV may eliminate the need for insufflation thereby mitigating the hemodynamics effects. For abdominal procedures, the IAP should be kept to less than $8-12 \mathrm{~cm} \mathrm{H}_{2} \mathrm{O}$ to limit the potential for adverse hemodynamic effects especially in patients with compromised myocardial function.

Despite its prevalence, there are limited previous reports regarding the perioperative care of patients with HCM [13, 29-35]. The reported literature has demonstrated success with various intravenous induction agents including midazolam, propofol, barbiturates (thiopentone), and etomidate. Maintenance anesthesia has included opioids (fentanyl, morphine and remifentanil) in combination with halothane, sevoflurane or isoflurane with or without nitrous oxide, and occasional supplementation with dexmedetomidine. In an effort to avoid or limit the need for general anesthesia, regional anesthesia including peripheral and neuraxial blockade has been reported especially in the parturient. These reports demonstrate the basic tenets of caring for such patients including: 1) maintenance of sinus rhythm with control of heart rate; 2) reduction in sympathetic activity to reduce chronotropy and inotropy; 3 ) maintenance of left ventricular filling pressures (preload); 4) control of variables responsible for myocardial oxygen delivery and demand; 5) maintenance of normal myocardial contractility; and 6) preservation of normal pulmonary vascular resistance.

\section{References}

1. Maron BJ, Ommen SR, Semsarian C, Spirito P, Olivotto I, Maron MS. Hypertrophic cardiomyopathy: present and future, with translation into contemporary cardiovascular medicine. J Am Coll Cardiol. 2014;64(1):83-99.

2. Maron BJ, Maron MS. Hypertrophic cardiomyopathy. Lancet. 2013;381(9862):242-255.

3. Teare D. Asymmetrical hypertrophy of the heart in young adults. Br Heart J. 1958;20(1):1-8.

4. Brock R. Functional obstruction of the left ventricle; acquired aortic subvalvar stenosis. Guys Hosp Rep. 1957;106(4):221-238.

5. Maron BJ, Gardin JM, Flack JM, Gidding SS, Kurosaki TT, Bild DE. Prevalence of hypertrophic cardiomyopathy in a general population of young adults. Echocardiographic analysis of 4111 subjects in the CARDIA Study. Coronary Artery Risk Development in (Young) Adults. Circulation. 1995;92(4):785-789.

6. Maron BJ. Cardiology patient pages. Hypertrophic cardiomyopathy. Circulation. 2002;106(19):2419-2421.

7. Maron BJ, Maron MS, Semsarian C. Genetics of hypertrophic cardiomyopathy after 20 years: clinical perspectives. J Am Coll Cardiol. 2012;60(8):705-715.

8. Ho CY. Genetics and clinical destiny: improving care in hypertrophic cardiomyopathy. Circulation. 2010;122(23):2430-2440; discussion 2440.

9. Richard P, Charron P, Carrier L, Ledeuil C, Cheav T, Pichereau C, Benaiche A, et al. Hypertrophic cardiomyopathy: distribution of disease genes, spectrum of mutations, and implications for a molecular diagnosis strategy.
Circulation. 2003;107(17):2227-2232.

10. Maron BJ, Seidman CE, Ackerman MJ, Towbin JA, Maron MS, Ommen SR, Nishimura RA, et al. How should hypertrophic cardiomyopathy be classified?: What's in a name? Dilemmas in nomenclature characterizing hypertrophic cardiomyopathy and left ventricular hypertrophy. Circ Cardiovasc Genet. 2009;2(1):81-85; discussion 86.

11. Maron BJ, Epstein SE. Hypertrophic cardiomyopathy: a discussion of nomenclature. Am J Cardiol. 1979;43(6):1242-1244.

12. Gajewski M, Hillel Z. Anesthesia management of patients with hypertrophic obstructive cardiomyopathy. Prog Cardiovasc Dis. 2012;54(6):503-511.

13. Sahoo RK, Dash SK, Raut PS, Badole UR, Upasani CB. Perioperative anesthetic management of patients with hypertrophic cardiomyopathy for noncardiac surgery: a case series. Ann Card Anaesth. 2010;13(3):253-256.

14. Haering JM, Comunale ME, Parker RA, Lowenstein E, Douglas PS, Krumholz HM, Manning WJ. Cardiac risk of noncardiac surgery in patients with asymmetric septal hypertrophy. Anesthesiology. 1996;85(2):254-259.

15. Tobias JD. Etomidate: applications in pediatric critical care and pediatric anesthesiology. Pediatr Crit Care Med. 2000;1(2):100-106.

16. Tobias JD. Etomidate in pediatric anesthesiology: Where are we now? Saudi J Anaesth. 2015;9(4):451-456.

17. Komatsu R, You J, Mascha EJ, Sessler DI, Kasuya Y, Turan A. Anesthetic induction with etomidate, rather than propofol, is associated with increased 30-day mortality and cardiovascular morbidity after noncardiac surgery. Anesth Analg. 2013;117(6):1329-1337.

18. Wagner CE, Bick JS, Johnson D, Ahmad R, Han X, Ehrenfeld JM, Schildcrout JS, et al. Etomidate use and postoperative outcomes among cardiac surgery patients. Anesthesiology. 2014;120(3):579-589.

19. Tobias JD, Gupta P, Naguib A, Yates AR. Dexmedetomidine: applications for the pediatric patient with congenital heart disease. Pediatr Cardiol. 2011;32(8):1075-1087.

20. Kernan S, Rehman S, Meyer T, Bourbeau J, Caron N, Tobias JD. Effects of dexmedetomidine on oxygenation during one-lung ventilation for thoracic surgery in adults. J Minim Access Surg. 2011;7(4):227-231.

21. Maron BJ, Wolfson JK, Epstein SE, Roberts WC. Intramural ("small vessel") coronary artery disease in hypertrophic cardiomyopathy. J Am Coll Cardiol. 1986;8(3):545557.

22. Vincent JL. Does central venous oxygen saturation accurately reflect mixed venous oxygen saturation? Nothing is simple, unfortunately. Intensive Care Med. 1992;18(7):386-387.

23. Della Rocca G, Pompei L. Goal-directed therapy in anesthesia: any clinical impact or just a fashion? Minerva Anestesiol. 2011;77(5):545-553.

24. Viale JP, Annat GJ, Ravat FM, Pommier CM, Brudon JR, Desuzinges CJ, Bertrand OM, et al. Oxygen uptake and mixed venous oxygen saturation during aortic surgery and the first three postoperative hours. Anesth Analg. 1991;73(5):530-535.

25. Bernard JM, Le Penven-Henninger C, Passuti N. Sud- 
den decreases in mixed venous oxygen saturation during posterior spinal fusion. Anesth Analg. 1995;80(5):10381041.

26. Tobias JD. Cerebral oxygenation monitoring: nearinfrared spectroscopy. Expert Rev Med Devices. 2006;3(2):235-243.

27. Tobias JD. Anesthetic considerations for laparoscopy in children. Semin Laparosc Surg. 1998;5(1):60-66.

28. Tobias JD. Anesthesia for minimally invasive surgery in children. Best Pract Res: Clin Anesth. 2002;12:272-277.

29. Jain A, Jain K, Bhagat H, Mangal K, Batra Y. Anesthetic management of a patient with hypertrophic obstructive cardiomyopathy with dual-chamber pacemaker undergoing transurethral resection of the prostate. Ann Card Anaesth. 2010;13(3):246-248.

30. Ahmed A, Zaidi RA, Hoda MQ, Ullah H. Anesthetic management of a patient with hypertrophic obstructive cardiomyopathy undergoing modified radical mastectomy. Middle East J Anaesthesiol. 2010;20(5):739-742.

31. Wadsworth R, Greer R, MacDonald JM, Vohra A. The use of remifentanil during general anaesthesia for caesarean delivery in two patients with severe heart dysfunction. Int J Obstet Anesth. 2002;11(1):38-43.

32. Rupal S, Swami AC, Jindal S, Lata S. Perioperative management of an elderly patient of hypertrophic obstructive cardiomyopathy for knee arthroplasty and the role of peripheral nerve blocks. Indian J Anaesth. 2013;57(4):394396.

33. Nama RK, Parikh GP, Patel HR. Anesthetic management of a patient with hypertrophic cardiomyopathy with atrial flutter posted for percutaneous nephrolithotomy. Anesth Essays Res. 2015;9(2):284-286.

34. Nikhar S, Yadav M, Gopinath R, Azharuddin M. Anesthetic management of carcinoma of thyroid involving trachea in a patient with hypertrophic obstructive cardiomyopathy. Ain-Shams J Anaesthesiol. 2015;8:144-147.

35. Harley ID, Jones EF, Liu G, McCall PR, McNicol PL. Orthotopic liver transplantation in two patients with hypertrophic obstructive cardiomyopathy. $\mathrm{Br} \mathrm{J}$ Anaesth. 1996;77(5):675-677. 